\title{
Some Variables Affecting the Recovery of Anaerobic Bacteria: a Quantitative Study
}

\author{
By B. WATT \\ Department of Bacteriology, Medical School, University of Edinburgh, EH8 $9 A G$ \\ MARGARET V. HOARE, \\ Department of Statistics, University of Edinburgh, 2I Buccleuch Place, EH8 ${ }_{9} L \mathrm{~N}$ \\ AND J. G. COLLEE \\ Department of Bacteriology, Medical School, University of Edinburgh, EH8 $9 A G$
}

(Received I3 February 1973; revised 25 March 1973)

SUMMARY

Quantitative studies with a demanding anaerobe (Clostridium oedematiens type D) have shown that colony counts of the organism obtained from plates incubated in a given Baird \& Tatlock (B.T.L.) anaerobic jar may differ markedly from the counts obtained with identical inocula on plates processed in an alternative jar of the same type. An investigation of jar variation led to improved experimental designs, so that statistically valid results could be obtained from quantitative studies. Such experiments showed the importance of multiple catalyst sachets for the recovery of organisms of the $C$. oedematiens group.

\section{INTRODUCTION}

An anaerobic jar incorporating a catalyst active at room temperature is marketed by Baird \& Tatlock Ltd. (B.T.L.). It was first described in 1958 (Lab. Equipment Test Report, I958) and is now widely used in Britain. Several workers have assessed the performance of similar equipment in the quantitative recovery of clostridia (e.g. Futter \& Richardson, I97I). Collee, Watt, Fowler \& Brown (1972) compared the quantitative recovery of anaerobes grown in B.T.L. jars when the jars were processed by a standard procedure and when they were used in conjunction with a disposable foil pack that liberates hydrogen and carbon dioxide ('Gaspak', Becton, Dickinson U.K. Ltd, Wembley, Middlesex).

Studies with anaerobic jars have generally been based on the assumption that the equipment behaves in a consistently reproducible manner. The present study shows that colony counts of an exacting anaerobe obtained on plates incubated in a given B.T.L. anaerobic jar can differ markedly from the counts obtained with identical inocula on plates processed in an alternative jar of the same type. As this observation is of importance in the design and interpretation of quantitative experiments involving anaerobic jars, the variables associated with the operation of the B.T.L. anaerobic jar were investigated. It was then possible to take account of these to produce experimental designs that are likely to give statistically valid results in quantitative studies with anaerobes. 


\section{METHODS}

Test strains. The following test strains were used in the present study: Clostridium oedematiens (C. novyi) type D, NCTC $8350 ; C$. tetani type VI, NCTC $9567 ; C$. oedematiens type A, NCTC 538; Bacteroides fragilis, NCTC 9343; and an anaerobic coccus (Hare's Group I), NCTC 980 .

All strains were subcultured in cooked-meat broth (see below). Their purity was checked by reference to colonial morphology, Gram-staining properties, and biochemical reactions. Duplicate plates seeded from the test cultures were incubated aerobically, to detect aerobic contamination, and anaerobically.

Media. Cooked-meat broth (CMB) was prepared as described by Cruickshank (1968) but the infusion broth component was made from Nutrient Broth no. 2 (Oxoid Ltd, London). Presteamed nutrient broth was used as a diluent; it was heated in a steamer at $100^{\circ} \mathrm{C}$ for $30 \mathrm{~min}$ to remove dissolved air, then cooled rapidly to $37^{\circ} \mathrm{C}$.

Equine blood agar (EBA) plates were prepared with Oxoid Blood Agar Base no. 2 enriched with equine blood (I0 \%, v/v). Cysteine-dithiothreitol equine blood agar (CDEBA) plates were prepared as described by Watt (1972a).

Blood. Defibrinated horse blood was supplied by Wellcome Laboratories (Wellcome Reagents Ltd, Wellcome Research Laboratories, Beckenham, Kent).

Anaerobic jars. Fourteen Baird \& Tatlock Ltd (Chadwell Heath, Essex, England) anaerobic jars were used in the present study. Five of the jars were unused before the present studies; the remainder were in current use, and are here referred to as 'used'.

The detailed procedure for the setting up of the anaerobic jars (hydrogen $90 \%$, carbon dioxide $10 \%$ ) was described by Collee, Rutter \& Watt, I97I. All jars were incubated at $37 \pm 0.5{ }^{\circ} \mathrm{C}$ (thermographically checked). In a given experiment, the period of incubation of all the jars was the same.

Catalyst sachets. Unless otherwise stated, each anaerobic jar was equipped with a single room-temperature catalyst sachet supplied by Baird \& Tatlock Ltd. Each sachet contained $20 \pm$ I catalytic pellets (mean of ten sachets examined).

Gases. Cylinders of carbon dioxide were supplied by the Distillers Company Ltd, Edinburgh. Cylinders of hydrogen, and of $90 \%$ hydrogen with $10 \%$ carbon dioxide, were supplied by the British Oxygen Company, London, SWI9 3 UF.

\section{Enumeration methods}

Total counts of bacteria and spores were performed as described by Collee et al. (1972) with a Hawksley counting chamber of $0.1 \mathrm{~mm}$ depth (BS 748). Viable counts were performed by a spread-plate method, with dilutions made in presteamed nutrient broth. Plates were seeded with $0.02 \mathrm{ml}$ inocula dropped from standard, calibrated Pasteur pipettes and spread with sterile glass spreaders of uniform size. Colony counts were calculated as the mean number of colonies grown on two or more plates from a given dilution; surface viable counts/ $\mathrm{ml}$ were derived from predetermined dilutions that gave Ioo to 700 evenly distributed colonies/plate.

Viable counts were recorded on a standard record sheet, and then transferred to punchcards for input to an IBM 360/50 computer at the Edinburgh Regional Computing Centre. All summary tables and analyses of variance were calculated by means of a program package developed at the Agricultural Research Council Unit of Statistics, Edinburgh. 
Table I. The colony counts observed on replicate plates each seeded from a dilution of an overnight CMB culture of Clostridium oedematiens type $D$ when the plates were incubated in I2 B.T.L. anaerobic jars

$\begin{array}{cc}\begin{array}{c}\text { Jars in } \\ \text { order of } \\ \text { evacuation }\end{array} & \begin{array}{c}\text { Condition* } \\ \text { of jar }\end{array} \\ \text { I } & \text { Used } \\ 2 & \text { New } \\ 3 & \text { New } \\ 4 & \text { Used } \\ 5 & \text { Used } \\ 6 & \text { New } \\ 7 & \text { Used } \\ 8 & \text { Used } \\ 9 & \text { New } \\ \text { I0 } & \text { Used } \\ \text { I I } & \text { Used } \\ \text { I2 } & \text { New } \dagger\end{array}$

No. of colonies subcultured from $4 \times 0.02 \mathrm{ml}$ of $\mathrm{CMB}$ culture at $10^{-2}$ dilution

\begin{tabular}{|c|c|c|c|}
\hline I I & 17 & 17 & 10 \\
\hline $2 I$ & 33 & $3 I$ & 30 \\
\hline I3I & 102 & 152 & 172 \\
\hline 3 & 3 & 8 & 2 \\
\hline 0 & 0 & 0 & 0 \\
\hline 34 & 43 & 34 & 23 \\
\hline 34 & 16 & I6 & 19 \\
\hline I I I & 85 & I 48 & 76 \\
\hline $7 \mathrm{I}$ & 92 & 57 & 63 \\
\hline 6I & 44 & $7 \mathrm{I}$ & 6I \\
\hline 6 & 4 & 2 & 5 \\
\hline 53 & 96 & $9 \mathrm{I}$ & $8 I$ \\
\hline
\end{tabular}

Secondary vacuum developed during initial processing after Io min at room temp. $(\mathrm{mmHg}$ below atmospheric pressure)

$$
\begin{aligned}
& -72 \\
& -60 \\
& -70 \\
& -64 \\
& -57 \\
& -48 \\
& -51 \\
& -24 \\
& -32 \\
& -52 \\
& -32
\end{aligned}
$$

* Used $=$ jar in current use; New = unused B.T.L. jar, used for first time in this experiment.

$\dagger$ No secondary vacuum was observed in this jar after $10 \mathrm{~min}$ and the catalyst was therefore replaced and the jar reprocessed.

All jars were processed according to the standard anaerobic procedure, and incubated in a single incubator at $37^{\circ} \mathrm{C}$ for $18 \mathrm{~h}$.

\section{RESULTS}

\section{Jar variation}

When plates of CDEBA medium seeded with identical inocula of Clostridium oedematiens type $D$ were incubated in different anaerobic jars, the colony counts varied widely. In a typical experiment, I 2 anaerobic jars (five 'unused' and seven 'used' - see Methods) were each equipped with a single unused room-temperature catalyst sachet. Forty-eight plates of freshly poured CDEBA medium, each seeded with $0.02 \mathrm{ml}$ of a $10^{-2}$ dilution of an overnight CMB culture of $C$. oedematiens type $\mathrm{D}$, were distributed without a formal randomization scheme between the $\mathrm{I} 2$ anaerobic jars, each jar receiving four plates. The jars were processed by a standard anaerobic procedure (Collee, Rutter \& Watt, 197I) in the order shown (Table I) and incubated at $37^{\circ} \mathrm{C}$ for I $8 \mathrm{~h}$. The observed recoveries on plates in different jars varied widely, and this variation primarily affected all the plates within a given jar, i.e. 'jar variation' was much more evident than 'plate variation'. Jar variation seemed to be unrelated to the history of the jar (used or unused) or to the actual degree of the secondary vacuum drawn by its active catalyst during the processing stage (but see below).

The regular occurrence of the jar variation phenomenon was investigated in a similar experiment with ten B.T.L. anaerobic jars on three successive days. Although large variations occurred between the mean number of colonies obtained per jar on a given day, the performance of a jar varied from day to day, i.e. a 'good' jar on one occasion might be a 'poor' jar on another occasion.

Several possible contributory factors are considered in the following series of quantitative experiments with the exacting Clostridium oedematiens type D in which vegetative cells were the significantly viable particles (Collee et al., 1971).

Effect of duration of exposure of Clostridium oedematiens type $D$ in diluent. A suitable dilution of an overnight CMB culture was held on the bench and used at ro min intervals 
to seed groups of replicate plates that were spread immediately, distributed between two anaerobic jars used for each Io min batch, and incubated immediately. After incubation, it was evident that the number of colonies decreased as the period of exposure to the diluent was increased. For example, the mean colony counts on 20 plates seeded and processed immediately in two jars were $496 \cdot 5 \pm 29 \cdot 6$ and $809 \cdot 5 \pm 33.4$. Identical inocula taken from the appropriate dilution bottle after Io min on the bench gave counts of 96 to I $30 /$ plate. Inocula taken at 60 min gave a mean count of $2.9 \pm 0.6$ for ro plates in one jar, and no growth was obtained on any of the Io plates in the other jar. A control plate included in the latter jar showed good growth.

Effect of duration of exposure to aerobic conditions. Results of similar experiments with seeded plates exposed at the bench to normal aerobic conditions indicated that the number of colonies decreased as the period of exposure of the seeded plates was increased prior to anaerobic processing. In a typical experiment, colony counts on plates processed and incubated immediately were in the range 240 to $250 /$ plate, whereas plates seeded with identical inocula and exposed to aerobic conditions for 90 min before processing showed no more than $o$ to 5 colonies.

Effect of potentially bacteriostatic substances in the anaerobic atmosphere. Possible factors considered included mercury from the manometer; suspensions of metallic salts, e.g. $\mathrm{CuSO}_{4}$, from the inside of the jar; and hydrogen sulphide $\left(\mathrm{H}_{2} \mathrm{~S}\right)$ and other possibly toxic factors evolved from solid or liquid cultures incubated in the same jar. Careful and prolonged studies with seeded plates exposed in anaerobic jars failed to reveal any consistent variability in colony counts due to these factors. For example, seeded plates incubated in jars lined with filter paper soaked with a saturated aqueous solution of $\mathrm{CuSO}_{4}$, or with water alone, continued to show the same variation of colony counts as control plates in normal jars. This was also true of plates incubated in jars containing a small amount of mercury, the accumulated scrapings from the interior surfaces of old jars, CMB cultures of various anaerobes, sterile CMB medium, or approximately $100 \mathrm{ml}$ of $\mathrm{H}_{2} \mathrm{~S}$ gas produced by reaction of $\mathrm{I} \mathrm{N}-\mathrm{HCl}$ with ferrous sulphide.

The effect of the age of the catalyst. Catalysts that had been used several times, but still showing satisfactory activity as judged by prompt production of a secondary vacuum, performed less well than new catalysts in terms of quantitative recovery of Clostridium oedematiens type D. In a series of experiments in which standard inocula were seeded on replicate plates, the highest mean colony counts were generally obtained with jars fitted with new catalyst sachets. For example mean counts of I3I5 and 722 were obtained on plates in two jars with new catalysts, compared with mean counts of I04, I88, 546 and 70 I on plates in four jars fitted with used catalysts. On another day, the respective counts with a new series of inocula were 393 and 424 compared with $324,334,357$ and 368 .

\section{Development of experimental design}

As no single factor primarily responsible for marked jar-to-jar variation was revealed by these studies, with the exception of the obvious roles of the catalyst sachet and the gas mixture, our experimental design for comparative studies was developed so that the comparisons under study would not be consistently biased, despite our inability to standardize all of the variables.

Each jar was cleaned, dried, coated with a silicone preparation ('Speedwax', Simoniz, London) and fitted with a new catalyst. All plates for a given experiment were poured from a single parent batch of medium in a uniform manner. The Petri dishes were of the plastic vented type (Sterilin Ltd, Richmond, Surrey). One test species or one test system, such as a 
Table 2. The influence of three variable factors on the recovery of Clostridium oedematiens type $D$ on solid medium

\begin{tabular}{|c|c|c|c|}
\hline Variable examined & Mean* & $\begin{array}{l}\text { Standard error } \\
\text { of the mean }\end{array}$ & $\begin{array}{l}\text { Significance of } \\
\text { the differencest }\end{array}$ \\
\hline \multicolumn{4}{|l|}{ No. of catalyst sachets } \\
\hline I & $18 \cdot 60)$ & \multirow{3}{*}{$0 \cdot 79$} & \multirow{3}{*}{$P<0.0 \mathrm{I}$} \\
\hline 3 & $22.55\}$ & & \\
\hline 5 & $23.53)$ & & \\
\hline \multicolumn{4}{|l|}{ Inoculum used $\ddagger$ on day } \\
\hline I & $17 \cdot 78\}$ & \multirow{3}{*}{0.79} & \multirow{3}{*}{$P<0.001$} \\
\hline 2 & $32 \cdot 46\}$ & & \\
\hline 3 & $14 \cdot 46$ & & \\
\hline \multicolumn{4}{|l|}{ Anaerobic jar used } \\
\hline I & $22 \cdot 87)$ & \multirow{6}{*}{$\mathrm{I} \cdot \mathrm{I} 2$} & \multirow{6}{*}{$P>0.05$} \\
\hline 2 & $2 I \cdot 8 I$ & & \\
\hline 3 & $2 \mathrm{I} \cdot 23$ & & \\
\hline 4 & $21 \cdot 88$ & & \\
\hline 5 & 20.99 & & \\
\hline 6 & $20 \cdot 59)$ & & \\
\hline
\end{tabular}

* The means and standard errors given are based on the square roots of the original colony counts. $\uparrow$ Significance levels are derived from the variance ratios obtained in the analysis of variance.

$\ddagger$ A different parent culture of the same strain incubated under identical conditions for the same period was used on each day; this accounts for the observed day-to-day differences in recovery.

faecal suspension, was involved. Dilutions were made in a standard way from a single batch of diluent, and all manipulations were done rapidly by a trained team, to minimize exposure to aerobic conditions. An experiment involving the preparation of dilutions from a parent culture, seeding and spreading of 60 plates, randomization and allocation to jars, and processing of six anaerobic jars occupied about $15 \mathrm{~min}$. The allocation of plates to jars, and their positions within the given jar were randomized by reference to randomization tables (Fisher \& Yates, I970), as was the order of processing of the jars. When experiments with a number of jars were performed over several days, a latin-square design was employed.

The influence of multiple catalyst sachets in B.T.L. jars on the surface growth of Clostridium oedematiens type $D$. Preliminary studies indicated that the recovery of $C$. oedematiens type $\mathrm{D}$ was increased when more than one catalyst sachet was used. This was confirmed in the following experiments.

Six B.T.L. jars were used on three separate occasions. Each jar was equipped with one, three or five new catalysts on day I, and the catalysts were redistributed on day 2 and day 3 , so that during the experiment each jar had all the possible permutations of numbers of catalysts.

On day I, 30 plates of freshly poured CDEBA medium were seeded from a $10^{-2}$ dilution of an overnight CMB culture. The plates were consecutively seeded in the order I to 30 , and each plate received a $0.02 \mathrm{ml}$ inoculum from the same dropping pipette. The plates were then randomly allocated to one of three anaerobic jars, and the position of plates within a given jar was also randomized. The jars were then processed in a predetermined random order. The process was repeated for the plates $3 \mathrm{I}$ to 60 . All jars were incubated for $18 \mathrm{~h}$ at $37^{\circ} \mathrm{C}$.

The experiment was repeated on days 2 and 3, a fresh $10^{-2}$ dilution of an overnight culture of the same test strain being used on each day. Fresh media (prepared from the same parent concentrate) were used on each day, with fresh supplements of cysteine-dithiothreitol (see 
Table 3. The influence of three variable factors on the recovery of Clostridium oedematiens type $A$ on solid medium

\begin{tabular}{|c|c|c|c|}
\hline Variable examined & Mean* & $\begin{array}{c}\text { Standard error } \\
\text { of the mean }\end{array}$ & $\begin{array}{l}\text { Significance of } \\
\text { the differences } \dagger\end{array}$ \\
\hline \multicolumn{4}{|l|}{ No. of catalyst sachets } \\
\hline $\mathbf{I}$ & $13.99)$ & \multirow{3}{*}{0.18} & \multirow{3}{*}{$P<0.01$} \\
\hline 3 & $15 \cdot 18\}$ & & \\
\hline 5 & $14 \cdot 83 J$ & & \\
\hline \multicolumn{4}{|l|}{ Inoculum used $\ddagger$ on day } \\
\hline $\mathbf{I}$ & $14 \cdot 00)$ & \multirow{3}{*}{0.18} & \multirow{3}{*}{$P<0.001$} \\
\hline 2 & 14.08 & & \\
\hline 3 & $15.91 \mathrm{I}$ & & \\
\hline \multicolumn{4}{|l|}{ Anaerobic jar used } \\
\hline $\mathbf{I}$ & $14.53)$ & \multirow{6}{*}{0.25} & \multirow{6}{*}{$P>0.05$} \\
\hline 2 & 14.92 & & \\
\hline 3 & 14.69 & & \\
\hline 4 & 1470 & & \\
\hline 5 & 14.13 & & \\
\hline 6 & $(5 \cdot 02)$ & & \\
\hline
\end{tabular}

* The means and standard errors given are based on the square roots of the original colony counts.

+ Significance levels are derived from the variance ratios obtained in the analysis of variance.

$\ddagger$ A different parent culture of the same strain incubated under identical conditions for the same period was used on each day; this accounts for the observed day-to-day differences in recovery.

Methods). As colony counts can be assumed to show a Poisson distribution (Meynell \& Meynell, 1970), the variance was stabilized by taking the square root of the original colony counts. An analysis of variance was then calculated (Armitage, 1971), and the results are summarized in Table 2. Higher colony counts were obtained in jars equipped with three or five catalysts than in those equipped with only one catalyst. Statistical analysis confirmed this but showed that there was no difference in recovery between jars with three or five catalyst sachets.

The influence of multiple catalyst sachets on the recovery of test anaerobes. The above experiment was repeated with the following organisms: Clostridium tetani type VI (NCTC 9569); Bacteroides fragilis (NCTC 9343); C. oedematiens type A (GRIA) and an anaerobic coccus (Hare's Group I) NCTC 9801.

The procedure followed was identical with that described above, except that equine blood agar (EBA) was used in place of CDEBA which does not enhance the growth of organisms other than those of the Clostridium oedematiens group (Watt, 1972a). Fresh randomizations were used for each organism on each day.

Statistical analysis of the results showed that for Clostridium tetani type VI, Bacteroides fragilis and the anaerobic coccus, no statistically significant differences were observed in the colony counts obtained with differing numbers of catalysts. In the case of $C$. oedematiens type A, however, analysis of the results (Table 3 ) showed that the number of catalyst sachets present in the jar significantly affected the magnitude of the colony counts $(P<0.0 \mathrm{I})$; although higher counts were obtained with three catalysts, there was no evidence that recovery was further increased by more than three catalysts/jar. With all organisms, the 'between jar' variation was not significant at the $5 \%$ level, although significant jar variation was encountered in other experiments with $C$. oedematiens type A.

The influence of multiple catalyst sachets on the recovery of anaerobic organisms from faeces. The recovery of total anaerobes from a faecal sample was determined in jars with 
one, three or five sachets, in a similar experiment to that described above, except that $0.02 \mathrm{ml}$ of a $1 \mathrm{O}^{-4}$ dilution of the sample was used on each day. Samples of the sample (diluted $\mathrm{I}$ in 20 in nutrient broth) were held deep frozen $\left(-20^{\circ} \mathrm{C}\right)$, to ensure reproducibility between days. The results were analysed as above and showed that there was no significant difference between the colony counts in jars with different numbers of catalysts and no evidence of significant jar variation was observed.

\section{DISCUSSION}

Although the B.T.L. anaerobic jar is widely used in service laboratories in this country and for quantitative studies with anaerobes (Futter \& Richardson, I97I), we know of no comparative investigations of the recovery of organisms from individual anaerobic jars. In the present study, there was considerable jar variation when an exacting organism such as Clostridium oedematiens type D was used. In our attempts to define the variables associated with the operation of the jar, many factors were relatively controlled; for example, checks on the size of the test inoculum showed that volumetric errors were very small compared with the degree of variation that we observed, and all experiments on a given day were performed with plates from the same batch of medium derived from a bulk concentrate. In any case, these factors were unlikely to affect all plates in a given jar and none in another jar. We were unable to demonstrate any inhibitory effects attributable to likely toxic factors that might be present in the atmosphere of the processed anaerobic jar. All plates in a given experiment were incubated for a fixed period of time at a constant temperature in a monitored incubator. Our standard anaerobic jar procedure ensured that variations in evacuation sequences and the composition of the final gas mixture were minimized. In statistically designed experiments, the jars had unused catalyst sachets attached in a standard fashion to the lids, because our preliminary studies indicated that old catalyst sachets performed less well than new catalysts with the $C$. oedematiens type-D model. Several factors such as exposure of the inoculum to aerobic conditions in diluents and on seeded plates clearly affected the recovery of $C$. oedematiens type D on solid media. A detailed experimental design involving randomization schedules then allowed us to take account of these variables so that we could make statistically valid comparisons of results obtained with different jars. The design took account of the following principles: (i) all plates were allocated to anaerobic jars according to a pre-arranged randomization schedule, and they were then distributed within a given jar according to a randomization scheme; (ii) the jars were processed in a random order and then incubated for equal periods at $37^{\circ} \mathrm{C}$; (iii) in experiments with demanding anaerobes at least four and preferably six replicate jars were used, and the experiment was repeated on several occasions.

Clearly, if jar variation can be shown to occur with anaerobes of direct clinical interest, then quantitative studies with these organisms will require careful design and analysis. So far we have been able to demonstrate jar variation on only one occasion with Clostridium tetani, but it occurred regularly with type A, B and D strains of $C$. oedematiens. Thus we are obliged to take account of this variation in the planning of future experiments. The negative findings in the recovery of anaerobes from faeces are important as a basis for further quantitative studies in which the B.T.L. anaerobic jar can be used with confidence provided that the procedures outlined above are followed.

Although previous workers have described experiments with two catalyst sachets in each of their anaerobic jars (Moore, I966; Walker, Harris \& Moore, 197I), the effect of multiple sachets on the recovery of anaerobes has not been adequately investigated. In the present 
study it was clear that the use of more than one sachet per jar significantly improved the recovery of organisms of the Clostridium oedematiens group, although little advantage accrued if more than three sachets were used. It may be that large numbers of individual catalytic pellets in a suitable container offer an acceptable alternative to the use of multiple catalyst sachets (T. A. Roberts, personal communication). The improved recovery has only been shown for the most exacting anaerobes that we tested. The negative findings in this series for organisms such as Bacteroides fragilis and $C$. tetani may indicate that we are already achieving satisfactory recovery with our standard anaerobic procedure; good recovery of anaerobes can certainly be achieved by conventional procedures (Watt, $1972 b$ ).

Further quantitative studies are clearly needed to define improved methods for the catalytic removal of oxygen from anaerobic jars. Until the results of such studies are available, the clinical microbiologist should seek to achieve reliable recovery of clinically important anaerobes by the use of B.T.L. anaerobic jars equipped with two or three catalyst sachets in conjunction with a standard anaerobic procedure.

The authors are indebted to $\mathrm{Mr} \mathrm{R}$. Brown for his skilled technical assistance and to Messrs Baird \& Tatlock Ltd for a generous supply of room-temperature catalyst sachets. The financial support of the Medical Research Council (Grant G 97I/II3/B) is gratefully acknowledged.

\section{REFERENCES}

Armitage, P. (I97I). Statistical Methods in Medical Research, p. 239. Oxford and London: Blackwell.

COllee, J. G., Rutter, J. M. \& WATT, B. (I97I). The significantly viable particle: a study of the subculture of an exacting sporing anaerobe. Journal of Medical Microbiology 4, 271-288.

Collee, J. G., WatT, B., Fowler, E. \& Brown, R. (1972). An evaluation of the Gaspak system in the culture of anaerobic bacteria. Journal of Applied Bacteriology 35, 71-82.

Cruickshank, R. (I968). Medical Microbiology, I I th edn (revised reprint), p. 757. Edinburgh and London: E. \& S. Livingstone.

Fisher, R. A. \& YATES, F. (1970). Statistical Tables for Biological, Agricultural and Medical Research, 6th edn. Edinburgh and London: Oliver \& Boyd.

FutTER, B. V. \& RichaRdson, G. (1971). Anaerobic jars in the quantitative recovery of clostridia. In Isolation of Anaerobes, p. 93. Edited by D. A. Shapton and R. G. Board. London and New York: Academic Press.

LAB EQuipment TeSt RePort No. 28 (1958). A new anaerobic jar. Laboratory Practice 7, 145-I46.

Meynell, G. G. \& Meynell, E. (I970). Theory and Practice in Experimental Bacteriology, 2nd edn. Cambridge University Press.

Moore, W. E. C. (I966). Techniques for routine culture of fastidious anaerobes. International Journal of Systematic Bacteriology 16, I73-190.

Walker, P. D., Harris, E. \& MoOre, W. B. (1971). The isolation of clostridia from animal tissues. In Isolation of Anaerobes, Ist edn, pp. 25-38. Edited by D. A. Shapton and R. G. Board. London and New York: Academic Press.

WATT, B. $(1972 a)$. The recovery of clinically important anaerobes on solid media. Journal of Medical Microbiology 5, 2 I I-2 18.

WATT, B. (1972 b). Studies in bacterial anaerobiosis: the recovery of clinically important anaerobes on solid media. M.D. Thesis, University of Edinburgh. 